\title{
Impact Of Quarry Effluent Discharge On Heavy Metal, Chlorophyll, Vitamin And Proximate Composition Of Selected Vegetables From Ishiagu Ebonyi State, Nigeria
}

\author{
${ }^{1,}$ Osuocha Kelechi U, ${ }^{2}$ Okereke Stanley C., ${ }^{3,}$ Ezekwe Ahamefula S, \\ ${ }^{4,}$ Chukwudouro Chieme., ${ }^{5,}$ Chukwu Ezinne C. \\ 1,2,3,4,5,(Department of Biochemistry/Abia State University, Uturu, Nigeria.)
}

\begin{abstract}
Heavy metal, chlorophyll, vitamin and proximate composition of selected vegetables in Ishiagu, Ebonyi State Nigeria as influenced by quarry effluent discharge were screened. Fresh samples of vegetables were collected from farms around Ishiagu quarry sites. Heavy metals were analyzed using Atomic Absorption Spectrophometer (ASS) while standard analytical methods were used to analyze other parameters. Results showed that lead concentration ranged from $0.0020 \pm 000^{a}-0.019 \pm 0.00^{a}$ in the test samples while the other heavy metals also showed significant increase in all the plants when compared to control $p<0.05$.Result also showed a significant decrease in chloropyll, chloropyll $_{b}$ and Total chlorophyll of the test samples analysed when compared to control.
\end{abstract}

KEY WORDS: Contamination, discharge, quarry, effluent, heavy metals

\section{INTRODUCTION}

The contamination of agricultural soil by quarry mining effluents usually alters the soil quality in terms of soil nutrient as soil is very important to human existence. Mining process creates a potentially negative impact in the environment both during the mining operations and for years after the mine is closed. These mining companies usually discharge the quarry effluent into nearby farms and these farm lands are cultivated by the rural dwellers.[1-3 ] reported that intake of heavy metal contaminated vegetables may pose a risk to human health. Rajesh et al [4 ] reported that intake of heavy metals are non-biodegradable and are persistent environmental contaminants. Vegetables are the edible parts of plant that are consumed wholly or in parts, raw or cooked as part of main dish or salad. Vegetables are rich sources of vitamins, minerals and fibers and also have beneficial oxidative effect. Iheanacho and Udebuani [5] reported that leafy vegetables are also sources of macro and micronutrients that play major roles in maintaining healthy living. Plants take up heavy metals by absorbing them from deposits on the part of the plants exposed to air from polluted environment as well as from contaminated soils[6-9]. Osuocha et al., [10] reported that excessive concentration of heavy metals in plants can cause oxidative stress, stomatal resistance, affect photosynthesis and chlorophyll florescence process. Vegetable plants growing on heavy metal contaminated medium can accumulate high concentrations of trace elements to cause serious health risk to consumers.

These heavy metals accumulate in these vegetables grown in these quarry effluent discharge farms which may ultimately go into human body through consumption of these vegetables. Jarup, [11] reported that prolonged consumption of unsafe level of these heavy metals through foodstuffs may lead to chronic accumulation of heavy metals in the kidney and liver of humans causing disruption of numerous biochemical processes, leading to cardiovascular, nervous, kidney and bone diseases. Mepha and Eboh [12] reported that leafy Vegetables are important source of both digestible and indigestible carbohydrate. They are also good sources of Vitamin C, Beta carotene and other nutrients and are responsible for more subtle feelings of daily well-being and for protection from long-term degenerative disease [13]. Leafy vegetables are important item of diet in many Nigerian homes. They are valuable sources of nutrient especially in rural areas where they contributed substantially to protein, minerals, vitamins, fibers and other nutrients which are usually in short supply in daily diet [14].The vegetables analyzed were based on the most commonly consumed vegetable in Ishiagu Ebonyi state Nigeria and includes

Telfaria occidentialis (fluted pumpkin) belongs to the family of plants called cucurbitaceae and is mostly cultivated for its nutritious leaves used as vegetables. Aletor et al.,[15] reported that in recent years fluted pumpkin has gained medicinal recognition and has been discovered to be blood purifiers and could therefore be useful in maintenance of good health. 
Garden egg leaf: Egg plant is botanically called Solanum melongena. The leaves are usually used in preparation of certain delicacies and belongs to the family Solanaceac and is widely distributed in the temperate and tropical regions. Garden egg leaves have been reportedly used in the treatment of certain diseases. Pumpkin with botanical name Cucurbita pepo is a herbaceous running plant belonging to the family Cucurbitacae and is one of the natural resources that grow well in the south eastern Nigeria. The popularly called "ugbogulu" plant is characterized by the presence of large and bristly leaves. The pulp is cooked and consume as food in many parts of Nigeria and also used as medication for deworming the intestine [16].Bitter leaf is popularly called Onugbu by the Igbos, ewuro by the Yorubas and shiwaka by the Hausa people. Venonia amygdalina is one of the leafy vegetables consumed in the south-eastern part of Nigeria and several medicinal values have been reported [17]

Amaranthus hybridus is a frost tender annual plant growing to $2 \mathrm{~m}$ (6ft $7 \mathrm{in}$ ) and belongs to the family Amaranthacae. It is spineless and grows up to $800 \mathrm{~m}$ high with grooves. The leaves are green, variable in shape and size. It is used in the treatment of intestinal bleeding, diarrhea and excessive menstruation. [5].Bearing in mind the possible impact of quarry effluent discharge on the immediate environment, there is need therefore to evaluate the heavy metal concentration of these vegetables grown in mining effluent contaminated soils and the possible impact of these effluent discharge on the nutritional assessment of these plants from Ishiagu Ebonyi State Nigeria as these quarry mining companies discharge their effluent into nearby farms which are cultivated by the rural settlers producing crops and vegetables not only for local consumption but for sales to other parts of the country.

\subsection{STUDY AREA}

\section{MATERIALS AND METHODS}

The study was carried out in Ishiagu, Ebonyi State, Nigeria. Ishiagu is made of Zinc and lead mining companies while farming is the major occupation of the rural settlers producing farm products like cassava, vegetables, yam etc. These firms discharge their effluent into nearby farms which are cultivated by these rural farmers producing crops and vegetable not only for local consumption but for food supply to other parts of the country.

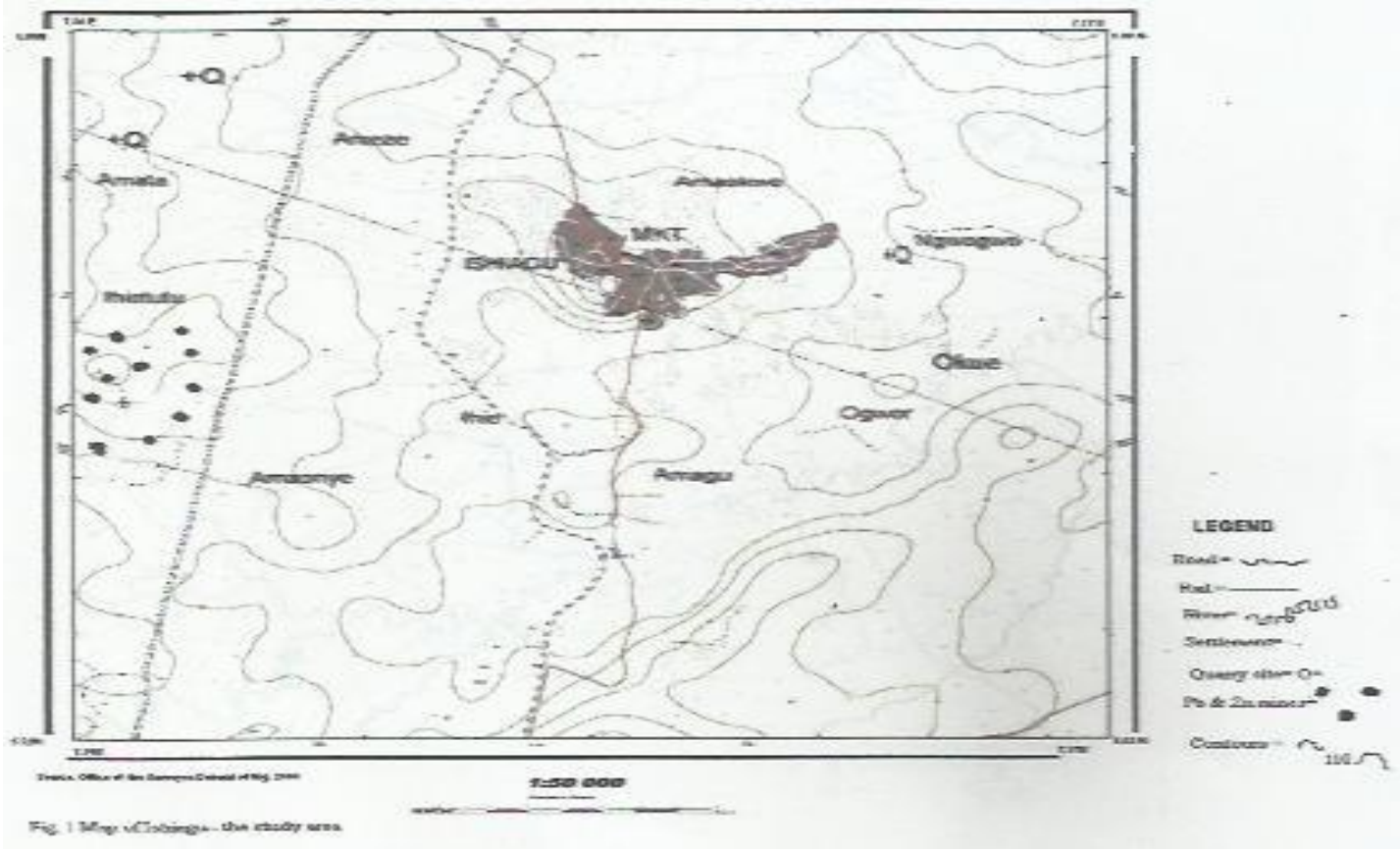

Map showing study area. Source [18]

\subsection{Sample collection and analysis}

The vegetables were obtained from farms around Ishiagu mining sites while the control samples were collected from unimpacted farms away from the mining areas. The samples for chlorophyll determination were immediately taken to Biochemistry laboratory, Abia state university, Uturu, Nigeria for immediate determination of the chlorophyll while the samples for heavy metals, vitamin content and proximate screening were oven dried at $60^{\circ} \mathrm{c}$ for further analysis. 


\subsection{Heavy metal analysis of the vegetables}

Heavy metal analysis of the samples were determined by the method of James, [19].

Following ashing of samples, the resulting ash was dissolved in $10 \mathrm{ml}$ of hydrochloric acid. It was filtered with whatman \#42 filter paper. The extract was used for analysis using Atomic Absorption Spectrophotometer (AAS).

\subsection{Chlorophyll Determination}

This was determined by the method described by Hendrick and Yvonne[20]. Exactly $0.15 \mathrm{~g}$ of fresh leaf was weighed into the mortar that was placed on ice. A bit of quartz sand was added to the mortar and ground until a homogenous mixture. $20 \mathrm{ml}$ of $80 \%$ acetone was added to the mixture. The suspension was quantitatively transferred to a centrifugation tube and centrifuged for about $5 \mathrm{~min}$ at maximum of $4500 \mathrm{rpm}$. The supernatant was transferred into a $25 \mathrm{ml}$ volumetric flask. The absorbance was read off using spectrophotometer at different wave lengths.

\subsection{Determination of vitamin content of selected vegetables.}

Vitamin A, C, E and B series in the vegetables were determined using the method of Association of Vitamin chemist as described by Kirk and Sawyer[21].

\subsection{Determination of proximate composition of the vegetables}

Moisture content, total ash, fiber content was determined by the method described by James[19], while protein and lipid contents were determined by the method described by Chang[22]. Carbohydrate content was done by the method of Change, [22].

\section{5 .Statistical analysis}

Data collected were subjected to statistical analysis using one way analysis of variance (ANOVA) procedure and difference in mean were separated $\mathrm{P}<0.05$ using standard students $\mathrm{t}-$ Test.

III. RESULTS AND DISCUSSION.

Table 1. shows heavy metal and mineral concentration $(\mathrm{mg} / \mathrm{kg})$ of selected vegetables from Ishiagu as influenced by quarry effluent discharge.

\begin{tabular}{|c|c|c|c|c|c|c|c|c|c|c|}
\hline Vegetable & \multicolumn{2}{|c|}{ Fluted pumpkin } & \multicolumn{2}{|c|}{ Wild green } & \multicolumn{2}{|c|}{ Bitterleaf } & \multicolumn{2}{|c|}{ Pumpkin } & \multicolumn{2}{|c|}{ Garden egg leaf } \\
\hline $\begin{array}{l}\text { Heavy } \\
\text { metals }\end{array}$ & A & B & A & B & A & B & A & B & A & B \\
\hline Lead & $\begin{array}{l}0.0200 \\
\pm \\
0.010^{\mathrm{a}}\end{array}$ & $\begin{array}{l}0.0040 \\
\pm \\
0.000^{\mathrm{a}}\end{array}$ & $\begin{array}{l}0.0020 \\
\pm \\
0.000^{\mathrm{a}}\end{array}$ & $\begin{array}{l}0.0020 \\
\pm \\
0.001^{\mathrm{a}}\end{array}$ & $\begin{array}{l}0.0620 \\
\pm \\
0.001^{\mathrm{a}}\end{array}$ & $\begin{array}{l}0.0960 \\
\pm \\
0.002^{\mathrm{b}}\end{array}$ & $\begin{array}{l}0.0190 \\
\pm \\
0.000^{\mathrm{a}}\end{array}$ & $\begin{array}{l}0.0730 \\
\pm \\
0.001^{\mathrm{b}}\end{array}$ & $\begin{array}{l}0.0270 \\
\pm \\
0.001^{\mathrm{a}}\end{array}$ & $\begin{array}{l}0.0620 \\
\pm \\
0.000^{\mathrm{b}}\end{array}$ \\
\hline Cadmium & $\begin{array}{l}0.0013 \\
\pm \\
0.001^{\mathrm{a}}\end{array}$ & $\begin{array}{l}0.0020 \\
\pm \\
0.000^{\mathrm{a}}\end{array}$ & $\begin{array}{l}0.0300 \\
\pm 0.010 \\
\mathrm{a}\end{array}$ & $\begin{array}{l}0.0500 \\
\pm \\
0.000^{\mathrm{b}}\end{array}$ & $\begin{array}{l}0.0130 \\
\pm \\
0.001^{\mathrm{a}}\end{array}$ & $\begin{array}{l}0.0340 \\
\pm \\
0.002^{\mathrm{b}}\end{array}$ & $\begin{array}{l}0.0090 \\
\pm \\
0.000^{\mathrm{a}}\end{array}$ & $\begin{array}{l}0.0260 \\
\pm \\
0.001^{b}\end{array}$ & $\begin{array}{l}0.0200 \\
\pm \\
0.001^{\mathrm{a}}\end{array}$ & $\begin{array}{l}0.0310 \\
\pm \\
0.000^{\mathrm{b}}\end{array}$ \\
\hline Chromium & $\begin{array}{l}0.0600 \\
\pm \\
0.010^{\mathrm{a}}\end{array}$ & $\begin{array}{l}0.0100 \\
\pm \\
0.000^{\mathrm{b}}\end{array}$ & $\begin{array}{l}0.0030 \\
\pm \\
0.001^{\mathrm{a}}\end{array}$ & $\begin{array}{l}0.0070 \\
\pm \\
0.001^{\mathrm{b}}\end{array}$ & $\begin{array}{l}0.0150 \\
\pm \\
0.001^{\mathrm{a}}\end{array}$ & $\begin{array}{l}0.0210 \\
\pm \\
0.001^{b}\end{array}$ & $\begin{array}{l}0.0030 \\
\pm \\
0.001^{\mathrm{a}}\end{array}$ & $\begin{array}{l}0.0510 \\
\pm \\
0.000^{\mathrm{b}}\end{array}$ & $\begin{array}{l}0.0540 \\
\pm \\
0.001^{\mathrm{a}}\end{array}$ & $\begin{array}{l}0.0530 \\
\pm \\
0.000^{\mathrm{a}}\end{array}$ \\
\hline Manganese & $\begin{array}{l}0.0110 \\
\pm \\
0.001^{\mathrm{a}}\end{array}$ & $\begin{array}{l}0.0313 \\
\pm \\
0.001^{\mathrm{b}}\end{array}$ & $\begin{array}{l}0.0127 \\
\pm \\
0.002^{\mathrm{a}}\end{array}$ & $\begin{array}{l}0.0113 \\
\pm \\
0.001^{\mathrm{a}}\end{array}$ & $\begin{array}{l}0.0090 \\
\pm \\
0.000^{\mathrm{a}}\end{array}$ & $\begin{array}{l}0.0120 \\
\pm \\
0.001^{\mathrm{b}}\end{array}$ & $\begin{array}{l}0.0270 \\
\pm \\
0.001^{\mathrm{a}}\end{array}$ & $\begin{array}{l}0.0530 \\
\pm \\
0.002^{\mathrm{b}}\end{array}$ & $\begin{array}{l}0.0370 \\
\pm \\
0.001^{\mathrm{a}}\end{array}$ & $\begin{array}{l}0.0413 \\
\pm \\
0.001^{\mathrm{b}}\end{array}$ \\
\hline Zinc & $\begin{array}{l}0.0300 \\
\pm \\
0.000^{\mathrm{a}}\end{array}$ & $\begin{array}{l}0.0833 \\
\pm \\
0.006^{b}\end{array}$ & $\begin{array}{l}0.0350 \\
\pm \\
0.001^{\mathrm{a}}\end{array}$ & $\begin{array}{l}0.0440 \\
\pm \\
0.002^{\mathrm{b}}\end{array}$ & $\begin{array}{l}0.0170 \\
\pm \\
0.001^{\mathrm{a}}\end{array}$ & $\begin{array}{l}0.0250 \\
\pm \\
0.001^{\mathrm{b}}\end{array}$ & $\begin{array}{l}0.0080 \\
\pm \\
0.001^{\mathrm{a}}\end{array}$ & $\begin{array}{l}0.0420 \\
\pm \\
0.001^{b}\end{array}$ & $\begin{array}{l}0.0040 \\
\pm \\
0.001^{\mathrm{a}}\end{array}$ & $\begin{array}{l}0.0280 \\
\pm \\
0.001^{\mathrm{b}}\end{array}$ \\
\hline Magnesium & $\begin{array}{l}0.0160 \\
\pm \\
0.001^{\mathrm{a}}\end{array}$ & $\begin{array}{l}0.0233 \\
\pm \\
0.001^{b}\end{array}$ & $\begin{array}{l}0.0630 \\
\pm \\
0.001^{\mathrm{a}}\end{array}$ & $\begin{array}{l}0.0710 \\
\pm \\
0.001^{b}\end{array}$ & $\begin{array}{l}0.0260 \\
\pm \\
0.001^{\mathrm{a}}\end{array}$ & $\begin{array}{l}0.0400 \\
\pm \\
0.000^{\mathrm{b}}\end{array}$ & $\begin{array}{l}0.0200 \\
\pm \\
0.000^{\mathrm{a}}\end{array}$ & $\begin{array}{l}0.0180 \\
\pm \\
0.001^{b}\end{array}$ & $\begin{array}{l}0.0280 \\
\pm \\
0.002^{\mathrm{a}}\end{array}$ & $\begin{array}{l}0.0320 \\
\pm \\
0.001^{\mathrm{b}}\end{array}$ \\
\hline Sodium & $\begin{array}{l}2.0700 \\
\pm \\
0.020^{\mathrm{a}}\end{array}$ & $\begin{array}{l}3.6700 \\
\pm \\
0.010^{\mathrm{b}}\end{array}$ & $\begin{array}{l}2.0900 \\
\pm \\
0.000^{\mathrm{a}}\end{array}$ & $\begin{array}{l}5.0100 \\
\pm \\
0.010^{\mathrm{b}}\end{array}$ & $\begin{array}{l}5.0100 \\
\pm \\
0.010^{\mathrm{a}}\end{array}$ & $\begin{array}{l}4.1000 \\
\pm \\
0.000^{\mathrm{b}}\end{array}$ & $\begin{array}{l}1.0600 \\
\pm \\
0.010^{\mathrm{a}}\end{array}$ & $\begin{array}{l}2.0900 \\
\pm \\
0.000^{\mathrm{b}}\end{array}$ & $\begin{array}{l}1.0400 \\
\pm \\
0.020^{\mathrm{a}}\end{array}$ & $\begin{array}{l}3.0200 \\
\pm \\
0.000^{\mathrm{b}}\end{array}$ \\
\hline Calcium & $\begin{array}{l}0.0600 \\
\pm \\
0.000^{\mathrm{a}}\end{array}$ & $\begin{array}{l}0.0913 \\
\pm \\
0.001^{b}\end{array}$ & $\begin{array}{l}0.0330 \\
\pm \\
0.002^{\mathrm{a}}\end{array}$ & $\begin{array}{l}0.0450 \\
\pm \\
0.001^{b}\end{array}$ & $\begin{array}{l}0.0100 \\
\pm \\
0.000^{\mathrm{a}}\end{array}$ & $\begin{array}{l}0.0270 \\
\pm \\
0.001^{\mathrm{b}}\end{array}$ & $\begin{array}{l}0.0060 \\
\pm \\
0.001^{\mathrm{a}}\end{array}$ & $\begin{array}{l}0.0313 \\
\pm \\
0.001^{b}\end{array}$ & $\begin{array}{l}0.0100 \\
\pm \\
0.000^{\mathrm{a}}\end{array}$ & $\begin{array}{l}0.0213 \\
\pm \\
0.001^{\mathrm{b}}\end{array}$ \\
\hline
\end{tabular}

Values are mean of triplicate determination \pm standard deviation.

Mean in the same row, having different alphabet are statistically significant $(\mathrm{p}<0.05)$. 
N/B. $A=$ Control vegetables from uncontaminated farms. $B=$ sample vegetables from quarry effluent contaminated farms.

Table 1 shows the concentration of heavy metals and minerals $(\mathrm{mg} / \mathrm{kg})$ of selected vegetables from Ishiagu as influenced by quarry effluent discharge. Results showed that heavy metals were more accumulated in plants grown around the mining sites than the control. This could be attributed to the fact that plants needs nutrients for survival and when these nutrients are not available due to heavy metal contamination they tend to absorb these metals more. All the plants absorbed substantial quantity of the heavy metals. This is in line with [23] who studied bioaccumulation of heavy metals grown in mining effluent contaminated soil treated with fertilizers. The toxicity of these heavy metals is well known but of major concern is possibility that continual exposure to relatively low level of these metals through consumption of these vegetable is of great concern as these heavy metals such as mercury and lead have been reported to cause liver, kidney disfunction[24].Table 2. shows chlorophyll $_{a}$, chlorophyll $\mathrm{b}_{\mathrm{b}}$ and total chlorophyll concentration $(\mathrm{mg} / \mathrm{ml})$ of selected vegetables from Ishiagu as influenced by quarry effluent discharge.

\begin{tabular}{|c|c|c|c|c|c|c|c|c|c|c|}
\hline Vegetable & \multicolumn{2}{|c|}{ Fluted pumpkin } & \multicolumn{2}{|c|}{ Wild green } & \multicolumn{2}{|c|}{ Bitterleaf } & \multicolumn{2}{|c|}{ Pumpkin } & \multicolumn{2}{|c|}{ Garden egg leaf } \\
\hline chlorophyll & $\mathrm{A}$ & $\mathrm{B}$ & A & $\mathrm{B}$ & A & B & $\mathrm{A}$ & $\mathrm{B}$ & A & B \\
\hline Chlorophyll $_{\mathrm{a}}$ & $\begin{array}{l}12.15 \\
\pm 0.01^{\mathrm{a}}\end{array}$ & $\begin{array}{l}11.25 \\
\pm 0.01^{\mathrm{b}}\end{array}$ & $\begin{array}{l}8.74 \pm \\
0.02^{\mathrm{a}}\end{array}$ & $\begin{array}{l}6.15 \pm \\
0.01^{\mathrm{b}}\end{array}$ & $\begin{array}{l}5.83 \pm \\
0.00^{\mathrm{a}}\end{array}$ & $\begin{array}{l}5.16 \pm \\
0.01^{\mathrm{b}}\end{array}$ & $\begin{array}{l}12.06 \\
\pm \\
0.01^{\mathrm{a}}\end{array}$ & $\begin{array}{l}6.04 \pm \\
0.00^{\mathrm{b}}\end{array}$ & $\begin{array}{l}8.71 \pm \\
0.25^{\mathrm{a}}\end{array}$ & $\begin{array}{l}8.20 \pm \\
0.00^{\mathrm{b}}\end{array}$ \\
\hline Chlorophyll $\mathrm{b}_{\mathrm{b}}$ & $\begin{array}{l}15.60 \\
\pm 0.00^{\mathrm{a}}\end{array}$ & $\begin{array}{l}9.25 \pm \\
0.02^{\mathrm{b}}\end{array}$ & $\begin{array}{l}9.41 \pm \\
0.02^{\mathrm{a}}\end{array}$ & $\begin{array}{l}8.09 \pm \\
0.02^{\mathrm{b}}\end{array}$ & $\begin{array}{l}7.26 \pm \\
0.04^{\mathrm{a}}\end{array}$ & $\begin{array}{l}6.73 \pm \\
0.01^{\mathrm{b}}\end{array}$ & $\begin{array}{l}14.14 \\
\pm \\
0.03^{\mathrm{a}}\end{array}$ & $\begin{array}{l}14.17 \\
\pm \\
0.01^{\mathrm{a}}\end{array}$ & $\begin{array}{l}10.83 \\
\pm \\
0.00^{\mathrm{a}}\end{array}$ & $\begin{array}{l}10.76 \\
\pm \\
0.02^{b}\end{array}$ \\
\hline $\begin{array}{l}\text { Total } \\
\text { chlorophylll }\end{array}$ & $\begin{array}{l}38.56 \\
\pm 0.02^{\mathrm{a}}\end{array}$ & $\begin{array}{l}24.33 \\
\pm 0.24^{\mathrm{b}}\end{array}$ & $\begin{array}{l}26.74 \\
\pm \\
0.01^{\mathrm{a}}\end{array}$ & $\begin{array}{l}17.11 \\
\pm 0.02^{\mathrm{b}}\end{array}$ & $\begin{array}{l}23.04 \\
\pm \\
0.02^{\mathrm{a}}\end{array}$ & $\begin{array}{l}18.09 \\
\pm \\
0.00^{\mathrm{b}}\end{array}$ & $\begin{array}{l}35.36 \\
\pm \\
0.02^{\mathrm{a}}\end{array}$ & $\begin{array}{l}24.18 \\
\pm \\
0.02^{\mathrm{b}}\end{array}$ & $\begin{array}{l}27.57 \\
\pm \\
0.02^{\mathrm{a}}\end{array}$ & $\begin{array}{l}27.07 \\
\pm \\
0.01^{b}\end{array}$ \\
\hline
\end{tabular}

Values are mean of triplicate determination \pm standard deviation.

Mean in the same row, having different alphabet are statistically significant $(\mathrm{p}<0.05)$.

$\mathrm{N} / \mathrm{B}$. $\mathrm{A}=$ Control vegetables from uncontaminated farms.

$\mathrm{B}=$ sample vegetables from quarry effluent contaminated farms.

Table 2 shows chlorophyll $\mathrm{a}_{\mathrm{a}}$. Chloropyll $\mathrm{b}_{\mathrm{b}}$ and Total chlorophyll concentration $(\mathrm{mg} / \mathrm{ml})$ of selected vegetables from Ishiagu as influenced by quarry effluent discharge.Results showed significant decrease in both chloropyll ${ }_{\mathrm{a}}$, chloropyll $\mathrm{b}_{\mathrm{b}}$ and total chlorophyll content of samples when compared to control samples $\mathrm{p}<0.05$. Osuocha et al., [10] reported that these decline in chlorophyll content under heavy metal stress is believed to be due to inhibition of important enzymes $\alpha$-aminolevulic acid and protochlorophyllide reductase associated with chlorophyll biosynthesis. Similar decrease in chlorophyll under heavy metal stress was reported in Brassica Juncea L. [25], in sun flower [26], in Cucurbita pepo [10] and in almond[27].

Table 3 shows vitamin content $(\mathrm{mg} / 100 \mathrm{~g})$ of selected vegetables from Ishiagu as influenced by quarry effluent discharge

\begin{tabular}{|c|c|c|c|c|c|c|c|c|c|c|c|c|c|c|c|c|c|c|c|}
\hline Vegetable & Fluted & umpkin & & & Tild & reen & & & Bitte & leaf & & & Pur & kin & & Gar & , & egg leaf & \\
\hline Vitamins & A & B & & $\mathrm{A}$ & & B & & A & & B & & $\mathrm{A}$ & & B & & A & & B & \\
\hline Thiamine & $\begin{array}{l}15.10 \pm \\
0.10^{\mathrm{a}}\end{array}$ & $\begin{array}{l}10.02 \\
0.0^{\mathrm{b}}\end{array}$ & \pm & $\begin{array}{l}20.20 \\
0.10^{\mathrm{a}}\end{array}$ & \pm & $\begin{array}{l}16.01 \\
0.01^{b}\end{array}$ & \pm & $\begin{array}{l}4.60 \\
0.44^{\mathrm{a}}\end{array}$ & \pm & $\begin{array}{l}4.01 \\
0.01^{\mathrm{a}}\end{array}$ & \pm & $\begin{array}{l}2.40 \\
0.10^{\mathrm{a}}\end{array}$ & \pm & $\begin{array}{l}1.63 \\
0.06^{\mathrm{b}}\end{array}$ & \pm & $\begin{array}{l}2.80 \\
0.10^{\mathrm{a}}\end{array}$ & \pm & $\begin{array}{l}2.60 \\
0.10^{\mathrm{a}}\end{array}$ & \pm \\
\hline Riboflavin & $\begin{array}{l}1.00 \quad \pm \\
0.10^{\mathrm{a}}\end{array}$ & $\begin{array}{l}0.62 \\
0.10^{\mathrm{b}}\end{array}$ & \pm & $\begin{array}{l}1.60 \\
0.10^{\mathrm{a}}\end{array}$ & \pm & $\begin{array}{l}1.43 \\
0.06^{\mathrm{b}}\end{array}$ & \pm & $\begin{array}{l}1.70 \\
0.10^{\mathrm{a}}\end{array}$ & \pm & $\begin{array}{l}1.54 \\
0.01^{\mathrm{b}}\end{array}$ & \pm & $\begin{array}{l}0.87 \\
0.01^{\mathrm{a}}\end{array}$ & \pm & $\begin{array}{l}0.62 \\
0.01^{\mathrm{b}}\end{array}$ & \pm & $\begin{array}{l}0.68 \\
0.01^{\mathrm{a}}\end{array}$ & \pm & $\begin{array}{l}0.67 \\
0.01^{\mathrm{b}}\end{array}$ & \pm \\
\hline Niacin & $\begin{array}{l}0.50 \\
0.10^{\mathrm{a}}\end{array}$ & $\begin{array}{l}0.30 \\
0.10^{\mathrm{a}}\end{array}$ & \pm & $\begin{array}{l}0.04 \\
0.01^{\mathrm{a}}\end{array}$ & \pm & $\begin{array}{l}0.07 \\
0.01^{\mathrm{b}}\end{array}$ & \pm & $\begin{array}{l}0.50 \\
0.10^{\mathrm{a}}\end{array}$ & \pm & $\begin{array}{l}0.28 \\
0.36^{\mathrm{a}}\end{array}$ & \pm & $\begin{array}{l}0.60 \\
0.10^{\mathrm{a}}\end{array}$ & \pm & $\begin{array}{l}0.06 \\
0.01^{\mathrm{b}}\end{array}$ & \pm & $\begin{array}{l}0.78 \\
0.01^{\mathrm{a}}\end{array}$ & \pm & $\begin{array}{l}0.81 \\
0.01^{\mathrm{b}}\end{array}$ & \pm \\
\hline Ascorbic acid & $\begin{array}{l}5.11 \\
0.01^{\mathrm{a}}\end{array} \quad \pm$ & $\begin{array}{l}3.24 \\
0.01^{b}\end{array}$ & \pm & $\begin{array}{l}3.84 \\
0.01^{\mathrm{a}}\end{array}$ & \pm & $\begin{array}{l}3.74 \\
0.01^{b}\end{array}$ & \pm & $\begin{array}{l}1.68 \\
0.01^{\mathrm{a}}\end{array}$ & \pm & $\begin{array}{l}1.56 \\
0.01^{b}\end{array}$ & \pm & $\begin{array}{l}3.12 \\
0.01^{\mathrm{a}}\end{array}$ & \pm & $\begin{array}{l}2.96 \\
0.01^{b}\end{array}$ & \pm & $\begin{array}{l}2.40 \\
0.10^{\mathrm{a}}\end{array}$ & \pm & $\begin{array}{l}2.23 \\
0.01^{b}\end{array}$ & \pm \\
\hline Tocopherol & $\begin{array}{l}130.10 \pm \\
0.10^{\mathrm{a}}\end{array}$ & $\begin{array}{l}100.06 \\
0.0^{\mathrm{b}}\end{array}$ & \pm & $\begin{array}{l}66.40 \\
0.10^{\mathrm{a}}\end{array}$ & \pm & $\begin{array}{l}67.01 \\
0.01^{\mathrm{b}}\end{array}$ & \pm & $\begin{array}{l}31.63 \\
0.01^{\mathrm{a}}\end{array}$ & \pm & $\begin{array}{l}27.60 \\
0.10^{\mathrm{b}}\end{array}$ & \pm & $\begin{array}{l}61.60 \\
0.10^{\mathrm{a}}\end{array}$ & \pm & $\begin{array}{l}40.20 \\
0.10^{\mathrm{b}}\end{array}$ & \pm & $\begin{array}{l}50.10 \\
0.10^{\mathrm{a}}\end{array}$ & \pm & $\begin{array}{l}49.10 \\
0.10^{\mathrm{b}}\end{array}$ & \pm \\
\hline Carotene & $\begin{array}{l}276.53 \pm \\
0.06^{\mathrm{a}}\end{array}$ & $\begin{array}{l}256.40 \\
0.10^{\mathrm{b}}\end{array}$ & \pm & $\begin{array}{l}225.63 \\
0.01^{\mathrm{a}}\end{array}$ & \pm & $\begin{array}{l}220.70 \\
0.10^{\mathrm{b}}\end{array}$ & \pm & $\begin{array}{l}234.83 \\
0.01^{\mathrm{a}}\end{array}$ & \pm & $\begin{array}{l}201.30 \\
0.10^{\mathrm{b}}\end{array}$ & \pm & $\begin{array}{l}208.73 \\
0.01^{\mathrm{a}}\end{array}$ & \pm & $\begin{array}{l}196.50 \\
0.10^{\mathrm{b}}\end{array}$ & \pm & $\begin{array}{l}190.40 \\
0.10^{\mathrm{a}}\end{array}$ & \pm & $\begin{array}{l}190.10 \\
0.10^{\mathrm{a}}\end{array}$ & \pm \\
\hline
\end{tabular}

Values are mean of triplicate determination \pm standard deviation.

Mean in the same row, having different alphabet are statistically significant $(\mathrm{p}<0.05)$.

N/B. $A=$ Control vegetables from uncontaminated farms. $B=$ sample vegetables from quarry effluent contaminated farms 
Table 3.shows the vitamin content of selected vegetables from Ishiagu as influenced by quarry effluent discharge. The vitamin content of the control samples compared favorably with the values reported in previous work[28].Results showed that the effluent discharge impacted negatively on the vitamin content of the test samples analyzed. There is need therefore for proper discharge of these quarry effluent as results has shown its negative impact on the vitamin content of these vegetables. Table 4 shows proximate composition of selected vegetables in Ishiagu as influenced by quarry effluent discharged (\%)

\begin{tabular}{|c|c|c|c|c|c|c|c|c|c|c|}
\hline Vegetables & \multicolumn{2}{|c|}{ Fluted pumpkin } & \multicolumn{2}{|c|}{ Wild green } & \multicolumn{2}{|c|}{ Bitterleaf } & \multicolumn{2}{|c|}{ Pumpkin } & \multicolumn{2}{|c|}{ Garden egg leaf } \\
\hline $\begin{array}{l}\text { Proximate } \\
\text { composition }\end{array}$ & $\mathrm{A}$ & B & A & B & A & B & A & B & A & B \\
\hline Crude fiber & $\begin{array}{l}13.50 \\
\pm \\
0.01^{\text {a }}\end{array}$ & $\begin{array}{l}13.20 \\
\pm \\
0.02^{\mathrm{b}}\end{array}$ & $\begin{array}{l}9.50 \pm \\
0.10^{\mathrm{a}}\end{array}$ & $\begin{array}{l}9.20 \pm \\
0.10^{\mathrm{a}}\end{array}$ & $\begin{array}{l}11.10 \\
\pm \\
0.10^{\mathrm{a}}\end{array}$ & $\begin{array}{l}9.50 \pm \\
0.10^{\mathrm{b}}\end{array}$ & $\begin{array}{l}9.00 \pm \\
0.10^{\mathrm{a}}\end{array}$ & $\begin{array}{l}8.00 \pm \\
0.20^{\mathrm{b}}\end{array}$ & $\begin{array}{l}13.50 \\
\pm \\
0.10^{\mathrm{a}}\end{array}$ & $\begin{array}{l}7.50 \pm \\
5.37^{\mathrm{a}}\end{array}$ \\
\hline Ash & $\begin{array}{l}17.00 \\
\pm \\
0.10^{\mathrm{a}}\end{array}$ & $\begin{array}{l}16.75 \\
\pm \\
0.01^{b}\end{array}$ & $\begin{array}{l}19.00 \\
\pm \\
0.10^{\mathrm{a}}\end{array}$ & $\begin{array}{l}18.61 \\
\pm \\
0.00^{\mathrm{b}}\end{array}$ & $\begin{array}{l}16.50 \\
\pm \\
11.50^{\mathrm{a}}\end{array}$ & $\begin{array}{l}11.50 \\
\pm \\
0.20^{\mathrm{b}}\end{array}$ & $\begin{array}{l}18.50 \\
\pm \\
0.10^{\mathrm{a}}\end{array}$ & $\begin{array}{l}12.50 \\
\pm \\
0.20^{\mathrm{b}}\end{array}$ & $\begin{array}{l}22.50 \\
\pm \\
0.10^{\mathrm{a}}\end{array}$ & $\begin{array}{l}22.03 \\
\pm \\
0.00^{\mathrm{b}}\end{array}$ \\
\hline Moisture & $\begin{array}{l}6.00 \pm \\
0.10^{\mathrm{a}}\end{array}$ & $\begin{array}{l}5.79 \pm \\
0.02^{\mathrm{b}}\end{array}$ & $\begin{array}{l}15.00 \\
\pm \\
0.10^{\mathrm{a}}\end{array}$ & $\begin{array}{l}14.85 \\
\pm \\
0.02^{\mathrm{b}}\end{array}$ & $\begin{array}{l}6.50 \pm \\
0.10^{\mathrm{a}}\end{array}$ & $\begin{array}{l}6.00 \pm \\
0.20^{\mathrm{b}}\end{array}$ & $\begin{array}{l}6.50 \pm \\
0.10^{\mathrm{a}}\end{array}$ & $\begin{array}{l}8.00 \pm \\
0.00^{\mathrm{b}}\end{array}$ & $\begin{array}{l}9.00 \pm \\
0.10^{\mathrm{a}}\end{array}$ & $\begin{array}{l}12.00 \\
\pm \\
0.00^{\mathrm{b}}\end{array}$ \\
\hline Lipid & $\begin{array}{l}8.40 \pm \\
0.01^{\mathrm{a}}\end{array}$ & $\begin{array}{l}8.12 \pm \\
0.02^{\mathrm{b}}\end{array}$ & $\begin{array}{l}9.00 \pm \\
0.10^{\mathrm{a}}\end{array}$ & $\begin{array}{l}8.19 \pm \\
0.02^{\mathrm{b}}\end{array}$ & $\begin{array}{l}4.04 \pm \\
0.01^{\mathrm{a}}\end{array}$ & $\begin{array}{l}16.30 \\
\pm \\
0.20^{\mathrm{b}}\end{array}$ & $\begin{array}{l}12.00 \\
\pm \\
0.10^{\mathrm{a}}\end{array}$ & $\begin{array}{l}9.98 \pm \\
0.00^{\mathrm{b}}\end{array}$ & $\begin{array}{l}11.01 \\
\pm \\
0.10^{\mathrm{a}}\end{array}$ & $\begin{array}{l}9.95 \pm \\
0.02^{\mathrm{b}}\end{array}$ \\
\hline Protein & $\begin{array}{l}18.80 \\
\pm \\
0.10^{\mathrm{a}}\end{array}$ & $\begin{array}{l}17.23 \\
\pm \\
0.20^{\mathrm{b}}\end{array}$ & $\begin{array}{l}17.93 \\
\pm \\
0.01^{\mathrm{a}}\end{array}$ & $\begin{array}{l}17.50 \\
\pm \\
0.01^{\mathrm{b}}\end{array}$ & $\begin{array}{l}18.11 \\
\pm \\
0.01^{\mathrm{a}}\end{array}$ & $\begin{array}{l}17.10 \\
\pm \\
0.02^{\mathrm{b}}\end{array}$ & $\begin{array}{l}19.42 \\
\pm \\
0.01^{\mathrm{a}}\end{array}$ & $\begin{array}{l}19.25 \\
\pm \\
0.01^{\mathrm{b}}\end{array}$ & $\begin{array}{l}18.94 \\
\pm \\
0.01^{\mathrm{a}}\end{array}$ & $\begin{array}{l}18.03 \\
\pm \\
0.02^{\mathrm{b}}\end{array}$ \\
\hline Carbohydrate & $\begin{array}{l}36.30 \\
\pm \\
0.01^{\mathrm{a}}\end{array}$ & $\begin{array}{l}38.91 \\
\pm \\
0.00^{\mathrm{b}}\end{array}$ & $\begin{array}{l}29.57 \\
\pm \\
0.01^{\mathrm{a}}\end{array}$ & $\begin{array}{l}31.65 \\
\pm \\
0.00^{\mathrm{b}}\end{array}$ & $\begin{array}{l}43.85 \\
\pm \\
0.00^{\mathrm{a}}\end{array}$ & $\begin{array}{l}39.60 \\
\pm \\
0.06^{\mathrm{b}}\end{array}$ & $\begin{array}{l}34.58 \\
\pm \\
0.01^{\mathrm{a}}\end{array}$ & $\begin{array}{l}42.27 \\
\pm \\
0.01^{\mathrm{b}}\end{array}$ & $\begin{array}{l}25.05 \\
\pm \\
0.01^{\mathrm{a}}\end{array}$ & $\begin{array}{l}27.49 \\
\pm \\
0.00^{\mathrm{b}}\end{array}$ \\
\hline
\end{tabular}

Values are mean of triplicate determination \pm standard deviation.

Mean in the same row, having different alphabet are statistically significant $(\mathrm{p}<0.05)$.

N/B. A= Control vegetables from uncontaminated farms.

$\mathrm{B}=$ sample vegetables from quarry effluent contaminated farms

Table 4 shows the proximate composition of selected vegetables from Ishiagu as influenced by quarry effluent discharge. The results showed significant decrease in proximate composition of vegetables analyzed when compared to control samples. This is in accordance with Asaolu et al., [29] who evaluated proximate and mineral composition of Nigerian leafy vegetables. This shows that quarry effluent discharge impacted negatively on the nutritional composition of the vegetables. This also agreed with Osuocha et al.,[10] who examined the effect of mining effluent contaminated soil treated with fertilizers on growth, chlorophyll and proximate composition of Cucurbita pepo vegetable.

\section{CONCLUSION.}

Quarry effluent discharge eventually contaminates agricultural soils with heavy metals which are eventually taken up by plants. These heavy metals are toxic but of a major concern is their toxicity as a result of constant consumption through consumption of vegetables which might lead consumers to heavy metal toxicity if bioaccumulation occurs due to regular consumption. There is need therefore to adopt proper disposal and remediation system in other to forestall exposure of these vegetables to quarry effluent discharge as these vegetables eventually accumulate the heavy metals which may go into the human body via consumption of these vegetable.

\section{REFERENCES}

[1] G. Marshal, (2004). Enhancing food chain integrity: quality assurance mechanism for air pollution impacts on fruits and vegetables system. Crop post Harvest Programme. Final technical report (R7530) www.sussex.ac.uk/spru/1-4-7-1-11-1.html.

[2] M.A.Radwan, and A.K Salama. Market basket survey for some heavy metals in Egyptian fruits and vegetables. Food chemical toxicology. 44, 2006, 1273-1278

[3] X.Wang, T. Sato, B. Xing, and S. Tao. Health risk of heavy metals to the general public in Tianjan, China via consumption of vegetables and fish. Science Toxi. Environment. 350 (1-3), 2005, 28-37.

[4] K.S.Rajesh, A. Madhooloka , and M.M. Fiona,. Heavy metals in vegetables collected from production and market sites of a tropical urban area of India. Food and chemical toxicology. 47, 2009, 583-591. 
[5] K.Iheanacho. , and A.C Udebuani, Nutritional composition of some leafy vegetable consumed in Imo State, Nigeria. Journal of Applied Science and Environmental management. 13(3), 2009.

35-38.

[6] T. Khairiah, M.K Zalifah, Y.H Yin, and A. Aminath, The uptake of heavy by fruit type vegetables, grown in selected agricultural areas. Pak. J. Biol. Sci. 7(2), 2004 1438-1442.

[7] M.S Jassir, A. Shaker, and M.A Khaliq, Deposition of heavy metals on green leafy vegetables sold on roadsides of Riyadh city, Saudi Arabia. Bull Environ. Contam. Toxicol. 75, 2005 1020-1027.

[8] A.G Kachenko, and B Singh, Heavy metals contamination in vegetables grown in urban and metal smelter contaminated sites in Australia. Water Air Soil pollut. 169, 2006, 101-123.

[9] S. Singh, and M. Kumar, . Heavy metal load of soil. Water and vegetables in periurban Delhi. Environ. Monitor. Assess. 120, $200671-79$

[10] K.U .Osuocha, E.I Akubugwo, G.C. Chinyere, A.S Ezekwe, M.K Duru and L.A Nwaogu, Effects of mining effluent contaminated soil treated with fertilizers on growth parameters, chlorophyll and proximate composition of Cucurbita pepo vegetable. Journal of Biodiversity and Environmental Sciences.3(9),2013,1-8.

[11] L. Jarup, Hazards of heavy metal contamination. Br. Med. Bull. 68, 2003,167182.

[12] H.D. Mephba, L.Eboh, and E.B. Banigo, effect of processing treatments on the nutritive composition and consumer acceptance of some Nigerian edible leafy vegetables. African journal of food Agriculture nutrition and development. 7(1), 2007 23-26.

[13] G. Oboh, Effect of blanching on the antioxidant property of some tropical green leafy vegetable. Food Sci. Tech, 38, 2005, 513517

[14] M.I.Mohammed, and N.Sharif, (Mineral composition of some leafy vegetables consumed in Kano, Nigeria. Nigerian Journal of Basic and Applied Science, 19(2), 2011, 208-211.

[15] O. Aletor, A.A. Oshodi, and I. Ipinmoroti. Chemical composition of common leafy protein concentrate. Food chemistry, 78, 2002, 63-68.

[16] C.M Elinge, A. Muhammad, A.A. Siaka, A.S. Hannatu, I.J Peni, and Y. Yahaya. Nutritional and Antinutritional composition of pumpkin. Advances in food and Energy security. 2012, 22-28.

[17] A.R. Ejoh, A.N. Tanya, N.V. Djuikwo and C.M. Mbofung. Effect of processing and preservation methods on vitamin C and total carotinoid level of some veronia species. African journal of food, Agriculture, Nutrition and Development. 5(2), 2005, 1-5.

[18] V.O. Nwaugo, I.S. Onwuchekwa.C Ogbonna, and R.A Onyeagba. Assessment of Physicochemical and Biological Indices of Fluvial Deposits in Abandoned Mine Pits in Ishiagu, South Eastern Nigeria. Nigerian Journal of Microbiology. 23(1), 2009, 1830 1838

[19] C.S. James. Analytical chemistry of foods. blakie academic and professional. London. 505 - 5091995.

[20] P. Hendrik and Yvonne de Jong-Van Berkel. Determination of chlorophyll extraction. 2011.

[21] R. Kirk, and R.Sawyer. Pearson's composition and analysis of foods. Publ. Church HillLivingstone, Edinbburgh. 1998.

[22] S.K.C.Chang. Protein Analysis.3rd Edition, New York: Kluwer Academic/plenum publisher. 7-10. 2003.

[23] E.I. Akubugwo, K.U. Osuocha, L.A Nwaogu, and G.C Chinyere.Studies on bioaccumulation of heavy metals and selected minerals from mining effluent contaminated soil treated with fertilizers into Cucurbita pepo vegetable. Advances in Applied Science Research 4(4), 2013, 190-195.

[24] J.O.Duruibe,M.O.C Ogwuegbu and J.N Egwurugwu.Heavy metal pollution and human biotoxic effect.International Journal of Physical Sciences 2(5),2007,112-118.

[25] R. John,P.Ahmed K.Gadgil and S.Sharma. Heavy metal toxicity:effect on plant growth,biochemical parameters and metal accumulation by Brassica Juncia L. International Journal of plant production.3(3), 2009.

[26] F.K Zengin and O.Munzuroglu.Toxic effect of cadmium on metabolism of sunflower seedlings. Acta Agricul.Scand, Sect.B. Plant Soil Science 56,2006, 224-229.

[27] N, Ellomi, F.BeN, A. Rhouma, B. Ben, I. Mezghani and M. Boukhris. Cadmium induced growth inhibition and alteration of biochemical parameters in almond seedlings grown in solution culture. Acta Physiologiae Plantarum.29, 2007, 57-62

[28] H.M Wardlaw and M.Kessel.Prospertive in Nutrition. $5^{\text {th }}$ edition. Mc graw Hill Boston. 271-274, 2002.

[29] S.S. Asaolu, O.S. Adefemi, I.G. Oyakilome., Ajibulu K.E., and M.F.Asaolu. Proximate and mineral composition of Nigerian leafy Vegetables. Journal of Food Research 1(3), 2012, 214-591 